\title{
1 What is the test-retest reliability of common task-fMRI measures? New 2 empirical evidence and a meta-analysis
}

3

4
Maxwell L. Elliott ${ }^{1 \dagger}$, Annchen R. Knodt ${ }^{1 \dagger}$, David Ireland ${ }^{2}$, Meriwether L. Morris ${ }^{1}$, Richie Poulton ${ }^{2}$, Sandhya Ramrakha ${ }^{2}$, Maria L. Sison ${ }^{1}$, Terrie E. Moffitt ${ }^{1,3-5}$, Avshalom Caspi ${ }^{1,3-5}$, Ahmad R. Hariri $^{1 *}$

${ }^{1}$ Department of Psychology \& Neuroscience, Duke University, Box 104410, Durham, NC 27708, USA

${ }^{2}$ Dunedin Multidisciplinary Health and Development Research Unit, Department of Psychology, University of Otago, 163 Union St E, Dunedin, 9016, NZ

${ }^{3}$ Social, Genetic, \& Developmental Psychiatry Research Centre, Institute of Psychiatry, Psychology, \& Neuroscience, King's College London, De Crespigny Park, Denmark Hill, London SE5 $8 A F, U K$

${ }^{4}$ Department of Psychiatry \& Behavioral Sciences, Duke University School of Medicine, Durham, NC 27708, USA

${ }^{5}$ Center for Genomic and Computational Biology, Duke University Box 90338, Durham, NC 27708, USA

†These authors contributed equally to this work.

*Correspondence:

Ahmad R. Hariri, Ph.D.

Professor of Psychology and Neuroscience

Director, Laboratory of NeuroGenetics

Head, Cognition and Cognitive Neuroscience Training Program

Duke University

Durham, NC 27708, USA

Phone: (919) 684-8408

Email: ahmad.hariri@duke.edu

Running head: TASK-FMRI RELIABILITY NOVEL DATA AND META-ANALYSIS 


\section{Abstract}

39 Identifying brain biomarkers of disease risk is a growing priority in neuroscience. The ability to identify

40 meaningful biomarkers is limited by measurement reliability; unreliable measures are unsuitable for

41 predicting clinical outcomes. Measuring brain activity using task-fMRI is a major focus of biomarker

42 development; however, the reliability of task-fMRI has not been systematically evaluated. We present

43 converging evidence demonstrating poor reliability of task-fMRI measures. First, a meta-analysis of 90

44 experiments $(\mathrm{N}=1,008)$ revealed poor overall reliability (mean ICC=.397). Second, the test-retest

45 reliabilities of activity in a priori regions of interest across 11 common fMRI tasks collected in the context

46 of the Human Connectome Project $(\mathrm{N}=45)$ and the Dunedin Study $(\mathrm{N}=20)$ were poor (ICCs=.067-.485).

47 Collectively, these findings demonstrate that common task-fMRI measures are not currently suitable for

48 brain biomarker discovery or individual differences research. We review how this state of affairs came to

49 be and highlight avenues for improving task-fMRI reliability.

50 Key words: Neuroimaging, Individual Differences, Statistical Analysis, Cognitive Neuroscience 


\section{Introduction}

Since functional magnetic resonance imaging (fMRI) was introduced in 1992 (Kwong et al., 1992),

53 scientists have had unprecedented ability to non-invasively observe brain activity in behaving humans. In

54 conventional fMRI, regional brain activity is estimated by measuring the blood oxygen level-dependent

55 (BOLD) signal which indexes changes in blood oxygenation associated with neural activity (Logothetis et

56 al., 2001). One of the most common forms of BOLD fMRI is based on tasks during which researchers

57 "map" brain activity associated with specific cognitive functions by contrasting the regional BOLD signal

58 during a control condition with the BOLD signal during a condition of interest. In this way, task-fMRI has

59 given neuroscientists unique insights into the brain basis of human behavior, from basic perception to

60 complex thought, and has given clinicians and mental-health researchers the opportunity to directly measure

61 dysfunction in the organ responsible for disorder.

Originally, task-fMRI was primarily used to understand functions supported by the typical or

63 average human brain by measuring within-subject differences in activation between task and control

64 conditions, and averaging them together across subjects to measure a group effect. To this end, fMRI tasks

65 have been developed and optimized to elicit robust activation in a particular brain region of interest (ROI)

66 or circuit when specific experimental conditions are contrasted. For example, increased amygdala activity

67 is observed when subjects view emotional faces in comparison with geometric shapes and increased ventral

68 striatum activity is observed when subjects win money in comparison to when they lose money (Barch et

69 al., 2013). The robust brain activity elicited using this within-subjects approach led researchers to use the

70 same fMRI tasks to study between-subjects differences. The logic behind this strategy is straightforward:

71 if a brain region activates during a task, then individual differences in the magnitude of that activation may

72 contribute to individual differences in behavior as well as any associated risk for disorder. Thus, if the

73 amygdala is activated when people view threatening stimuli, then differences between people in the degree

74 of amygdala activation should signal differences between them in threat sensitivity and related clinical

75 phenomenon like anxiety and depression (Swartz et al., 2015). In this way, fMRI was transformed from a

76 tool for understanding how the average brain works to a tool for studying how the brains of individuals

77 differ. 
The use of task-fMRI to study differences between people heralded the possibility that it could

offer a powerful tool for discovering biomarkers for brain disorders (Woo et al., 2017). Broadly, a

80 biomarker is a biological indicator often used for risk stratification, diagnosis, prognosis and evaluation of

81 treatment response. However, to be useful as a biomarker, an indicator must first be reliable. Reliability is

82 the ability of a measure to give consistent results under similar circumstances. It puts a limit on the

83 predictive utility, power, and validity of any measure (see Box 1 and Fig. 1). In this way, reliability is

84 critical for both clinical applications and research practice. Measures with low reliability are unsuitable as

85 biomarkers and cannot predict clinical health outcomes. That is, if a measure is going to be used by

86 clinicians to predict the likelihood that a patient will develop an illness in the future, then the patient cannot

score randomly high on the measure at one assessment and low on the measure at the next assessment.
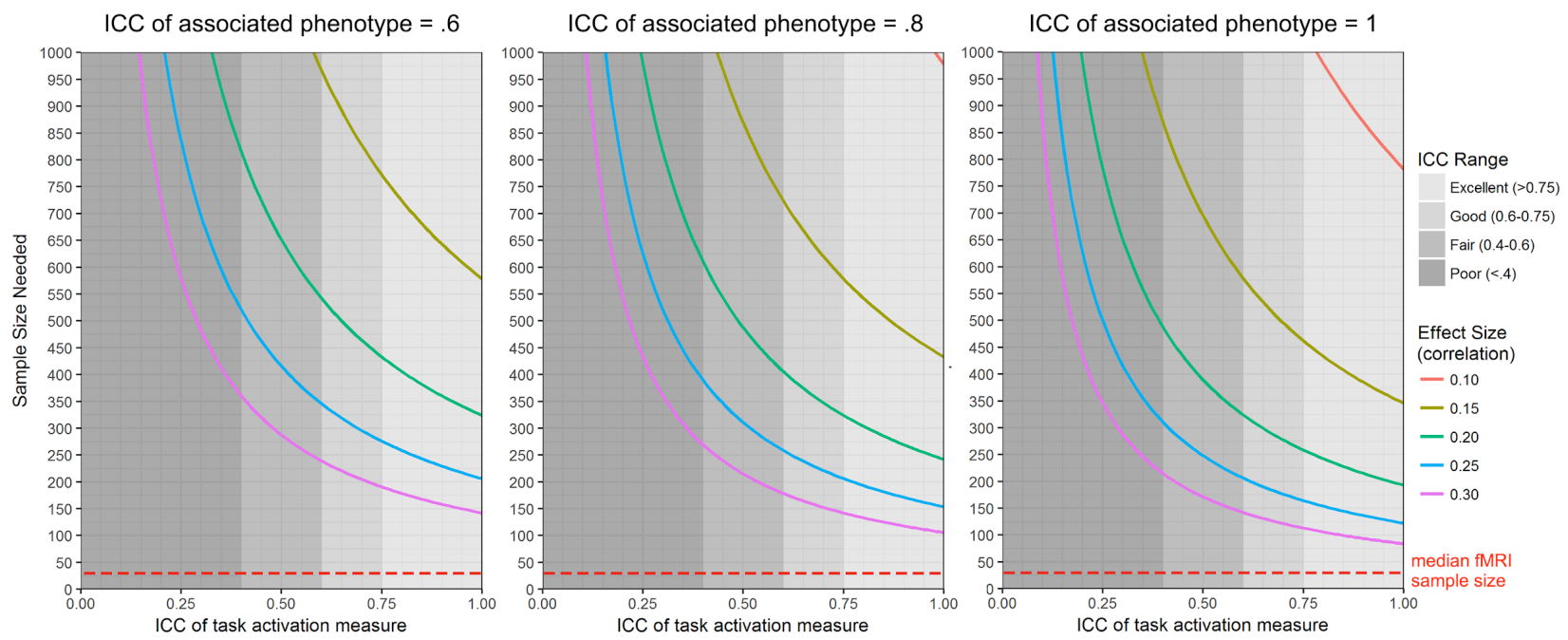

Fig. 1. The influence of task-fMRI test-retest reliability on sample size required for $80 \%$ power to detect brain-behavior correlations of effect sizes commonly found in psychological research. Power curves are calculated for three levels of reliability of the associated behavioral/clinical phenotype. The figure was generated using the "pwr.r.test" function in R, with the value for " $r$ " specified according to the attenuation formula in Box 1. The figure emphasizes the impact of low reliability at the lower $\mathrm{N}$ range because most fMRI studies are relatively small (median $\mathrm{N}=28.5$ (Poldrack et al., 2017)).

To progress toward a cumulative neuroscience of individual differences with clinical relevance we must establish reliable brain measures. While the reliability of task-fMRI has previously been discussed (Bennett \& Miller, 2010; Herting et al., 2018), individual studies provide highly variable estimates, often come from small test-retest samples employing a wide-variety of analytic methods, and sometimes reach 
contradictory conclusions about the reliability of the same tasks (Manuck et al., 2007; Nord et al., 2017).

102 This leaves the overall reliability of task-fMRI, as well as the specific reliabilities of many of the most

103 commonly used fMRI tasks, largely unknown. An up-to-date, comprehensive review and meta-analysis of

104 the reliability of task-fMRI and an in-depth examination of the reliability of the most widely used task-

105 fMRI measures is needed. Here, we present evidence from two lines of analysis that point to the poor

106 reliability of commonly used task-fMRI measures. First, we conducted a meta-analysis of the test-retest

107 reliability of regional activation in task-fMRI. Second, in two recently collected datasets, we conducted

108 pre-registered analyses (https://sites.google.com/site/moffittcaspiprojects/home/projectlist/knodt_2019) of

109 the test-retest reliability of brain activation in a priori regions of interest across several commonly used

110 fMRI tasks.

112 Methods

\section{Meta-analytic Reliability of Task-fMRI}

We performed a systematic review and meta-analysis following PRISMA guidelines (see

115 Supplemental Fig. S1). We searched Google Scholar for peer reviewed articles written in English and

116 published on or before April 1, 2019 that included test-retest reliability estimates of task-fMRI activation.

117 We used the advanced search tool to find articles that include all of the terms "ICC," "fmri," and "retest",

118 and at least one of the terms "ROI," "ROIs," "region of interest," or "regions of interest." This search yielded

1191,170 articles.

Study Selection and Data Extraction. One author (MLM) screened all titles and abstracts before

121 the full texts were reviewed (by authors MLE and ARK). We included all original, peer-reviewed empirical

122 articles that reported test-retest reliability estimates for activation during a BOLD fMRI task. All ICCs

123 reported in the main text and supplement were eligible for inclusion. If ICCs were only depicted graphically

124 (e.g. bar graph), we did our best at judging the value from the graph. Voxel-wise ICCs that were only 125 depicted on brain maps were not included. For ICCs calculated based on more than 2 time points, we used 126 the average of the intervals as the value for interval (e.g. the average of the time between time points 1 and 
1272 and time points 2 and 3 for an ICC based on 3 time points). For articles that reported ICCs from sensitivity

128 analyses in addition to primary analyses on the same data (e.g. using different modeling strategies or

129 excluding certain subjects) we only included ICCs from the primary analysis. We did not include ICCs

130 from combinations of tasks. ICCs were excluded if they were from a longitudinal or intervention study that

131 was designed to assess change, if they did not report ICCs based on measurements from the same MRI

132 scanner and/or task, or if they reported reliability on something other than activation measures across

133 subjects (e.g., spatial extent of activation or multi-voxel patterns of activation within subjects).

Two authors (MLE and ARK) extracted data about sample characteristics (publication year, sample

135 size, healthy versus clinical), study design (test-retest interval, event-related or blocked, task length, and

136 task type), and ICC reporting (i.e., was the ICC thresholded?). For each article, every reported ICC meeting

137 the above study-selection requirements was recorded.

Statistical Analyses. For most of the studies included, no standard error or confidence interval for

the ICC was reported. Therefore, in order to include as many estimates as possible in the meta-analysis, the

140 standard error of all ICCs was estimated using the Fisher r-to-Z transformation for ICC values (Chen et al.,

141 2018; McGraw \& Wong, 1996).

142 A random-effects multilevel meta-analytic model was fit using tools from the metafor package in

143 R (“Metafor Package R Code for Meta-Analysis Examples," 2019). In this model, ICCs and standard errors

144 were averaged within each unique sample, task, and test-retest interval (or "substudy") within each article

145 (or "study"; (Borenstein et al., 2009)). For the results reported in the Main Article, the correlation between

146 ICCs in each substudy was assumed to be 1 so as to ensure that the meta-analytic weight for each substudy

147 was based solely on sample size rather than the number of ICCs reported. However, sensitivity analyses

148 revealed that this decision had very little impact on the overall result (see Supplemental Fig. S2). In the

149 meta-analytic model, substudies were nested within studies to account for the non-independence of ICCs

150 estimated within the same study. Meta-analytic summaries were estimated separately for substudies that

151 reported ICC values that had been thresholded (i.e., when studies calculated multiple ICCs, but only 
152 reported values above a minimum threshold) because of the documented spurious inflation of effect sizes

153 that occur when only statistically significant estimates are reported (Kriegeskorte et al., 2009; Poldrack et

154 al., 2017; Vul et al., 2009; Yarkoni, 2009).

155 To test for effects of moderators, a separate random-effects multilevel model was fit to all 1,146

156 ICCs (i.e., without averaging within each substudy, since many substudies included ICCs with different

157 values for one or more moderators). The moderators included were task length, task design (block vs event-

158 related), task type (e.g. emotion, executive control, reward, etc), ROI type (e.g. structural or functional),

159 ROI location (cortical vs subcortical), sample type (healthy vs clinical), retest interval, number of citations

160 per year, and whether ICCs were thresholded on significance (see Supplemental Table S1 for descriptive

161 statistics on all moderators tested). All moderators were simultaneously entered into the model as random

162 effects. In the multi-level model, ICCs were nested within substudies, which were in turn nested within

163 studies. This was done to account for the non-independence of ICCs estimated within the same substudy,

164 as well as the non-independence of substudies conducted within the same study.

165

\section{Analyses of New Datasets}

Human Connectome Project (HCP). This is a publicly available dataset that includes 1,206

169 participants completed the entire scan protocol a second time (with a mean interval between scans of

170 approximately 140 days). All participants were free of current psychiatric or neurologic illness and were

171 between 25 and 35 years of age.

172 The seven tasks employed in the HCP were designed to identify functionally relevant "nodes" in

173 the brain. These tasks included an "n-back" working memory / executive function task (targeting the

174 dorsolateral prefrontal cortex, or dlPFC (Drobyshevsky et al., 2006)), a "gambling" reward / incentive

175 processing task (targeting the ventral striatum (Delgado et al., 2000)), a motor mapping task consisting of

176 foot, hand, and tongue movements (targeting the motor cortex (Drobyshevsky et al., 2006)), an auditory 
177 language task (targeting the anterior temporal lobe (Binder et al., 2011)), a social cognition / theory of mind

178 task (targeting the lateral fusiform gyrus, superior temporal sulcus, and other "social-network" regions

179 (Wheatley et al., 2007)), a relational processing / dimensional change detection task (targeting the

180 rostrolateral prefrontal cortex (R. Smith et al., 2007), or rlPFC), and a face-matching emotion processing

181 task (targeting the amygdala (Hariri et al., 2002)).

182 Dunedin Multidisciplinary Health and Development Study. The Dunedin Study is a longitudinal

183 investigation of health and behavior in a complete birth cohort of 1,037 individuals (91\% of eligible births;

$18452 \%$ male) born between April 1972 and March 1973 in Dunedin, New Zealand (NZ) and followed to age

18545 years (Poulton et al., 2015). Structural and functional neuroimaging data were collected between August

1862016 and April 2019, when participants were 45 years old. In addition, 20 Study members completed the

187 entire scan protocol a second time (with a mean interval between scans of 79 days).

Functional MRI was collected during four tasks targeting neural "hubs" in four different domains:

189 a face-matching emotion processing task (targeting the amygdala (Hariri et al., 2002)), a Stroop executive

190 function task (targeting the dlPFC and the dorsal anterior cingulate cortex (Peterson et al., 1999)), a

191 monetary incentive delay reward task (targeting the ventral striatum (Knutson et al., 2000)), and a face-

192 name encoding episodic memory task (targeting the hippocampus (Zeineh et al., 2003)). See Supplemental

193 Methods for additional details, including fMRI pre-processing, for both datasets.

194 ROI Definition. Individual estimates of regional brain activity were extracted according to two

195 commonly used approaches. First, we extracted average values from a priori anatomically defined regions.

196 We identified the primary region of interest (ROI) for each task and extracted average BOLD signal change

197 estimates from all voxels within a corresponding bilateral anatomical mask.

198 Second, we used functionally defined regions based on group-level activation. Here, we generated

199 functional ROIs by drawing $5 \mathrm{~mm}$ spheres around the group-level peak voxel within the target anatomical

200 ROI for each task (across all subjects and sessions). This is a commonly used strategy for capturing the

201 location of peak activation in each subject despite inter-subject variability in the exact location of the 
activation. See Supplemental Materials for further details on ROI definition, overlays on the anatomical template (Fig. S3), and peak voxel location (Table S2). We report analyses based on anatomically defined ROIs in the Main Article and report sensitivity analyses using functional ROIs in the Supplement.

Reliability Analysis. Subject-level BOLD signal change estimates were extracted for each task, ROI, and scanning session. Reliability was quantified using a 2-way mixed effects intraclass correlation coefficient (ICC), with session modeled as a fixed effect, subject as a random effect, and test-retest interval as an effect of no interest. This mixed effects model is referred to as ICC $(3,1)$ by Shrout and Fleiss (1979), and defined as:

$$
\operatorname{ICC}(3,1)=(B M S-E M S) /(B M S+(k-1) * E M S)
$$

212 "raters," or scanning sessions (in this case 2). We note that ICC $(3,1)$ tracks the consistency of measures

213 between sessions rather than absolute agreement, and is commonly used in studies of task-fMRI test-retest

214 reliability due to the possibility of habituation to the stimuli over time (Plichta et al., 2012).

219 patterns of reliability, we also calculated voxel-wise maps of ICC $(3,1)$ using AFNI's 3dICC_REML.R

220 function (Chen et al., 2013). Finally, to provide a benchmark for evaluating task-fMRI reliability, we

221 determined the test-retest reliability of three commonly used structural MRI measures: cortical thickness 222 and surface area for each of 360 parcels or ROIs (Glasser et al., 2016) as well as subcortical volume for 17 223 structures. These analyses were pre-registered

224 (https://sites.google.com/site/moffittcaspiprojects/home/projectlist/knodt_2019). Code and data for this 


\section{Reliability of Individual Differences in Task-fMRI: A Systematic Review and Meta-analysis}

233 per article and 5.7 citations per article, per year. During the study-selection process, we discovered that

234 some analyses calculated many different ICCs (across multiple ROIs, contrasts, and tasks), but only

235 reported a subset of the estimated ICCs that were either statistically significant or reached a minimum ICC

236 threshold. This practice leads to inflated reliability estimates (Kriegeskorte et al., 2010, 2009; Poldrack et

237 al., 2017). Therefore, we performed separate analyses of data from un-thresholded and thresholded reports.

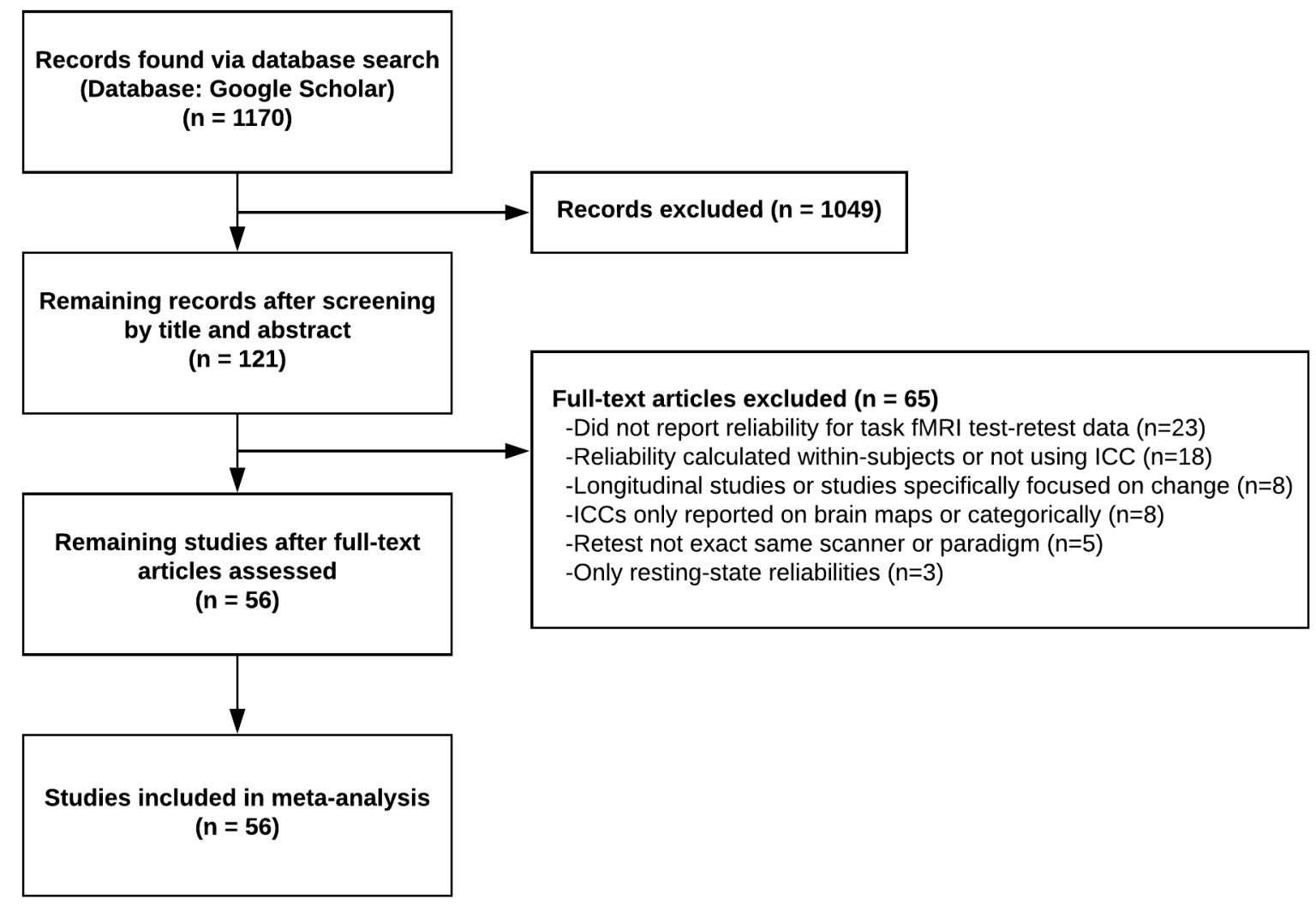

Fig. 2. Flow diagram for systematic literature review and meta-analysis. 

aCC-BY 4.0 International license.

Fig. 3 shows the test-retest reliability coefficients (ICCs) from 77 substudies reporting un-

243 thresholded values (average $\mathrm{N}=19.6$, median $\mathrm{N}=17$ ). $56 \%$ of the values fell into the range of what is

244 considered "poor" reliability (below .4), an additional $24 \%$ of the values fell into the range of what is

245 considered "fair" reliability (.4 - .6), and only 20\% fell into the range of what is considered "good" (.6 - .75)

246 or "excellent" (above .75) reliability. A random effects meta-analysis revealed an average ICC of .397 (95\%

247 CI, $.330-.460 ; \mathrm{P}<.001$ ), which is in the "poor" range (Cicchetti \& Sparrow, 1981). There was evidence

248 of between-study heterogeneity $\left(\mathrm{I}^{2}=31.6 ; \mathrm{P}=0.04\right)$. 
bioRxiv preprint doi: https://doi.org/10.1101/681700; this version posted January 16,2020 . The copyright holder for this preprint (which was not certified by peer review) is the author/funder, who has granted bioRxiv a license to display the preprint in perpetuity. It is made available under aCC-BY 4.0 International license.

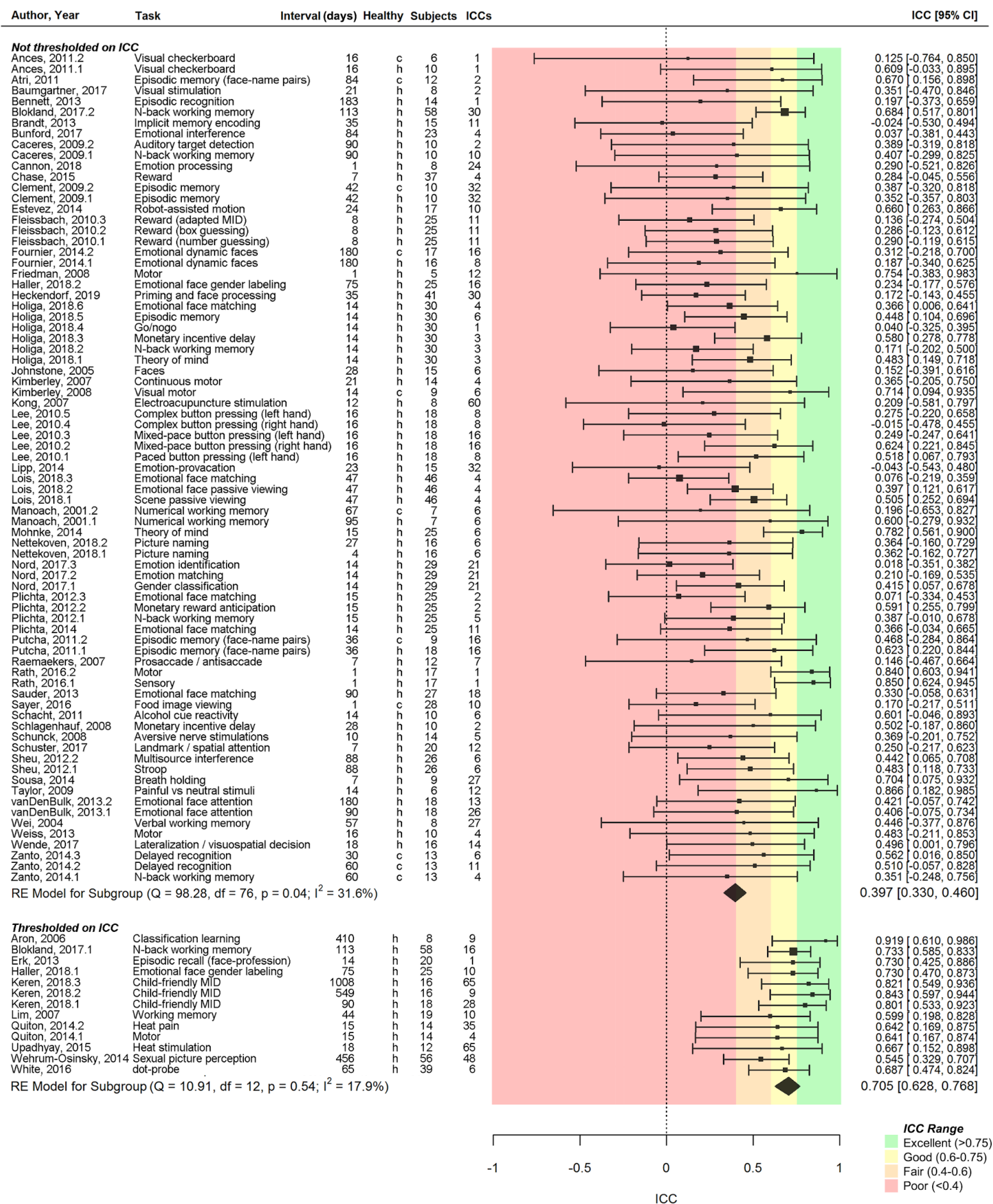

Fig. 3. Forest plot for the results of the meta-analysis of task-fMRI test-retest reliability. The forest plot displays the estimate of test-retest reliability of each task-fMRI measure from all ICCs reported in each study. Each substudy is labelled as h if the sample in the study consisted of healthy controls or $\mathrm{c}$ if the study consisted of a clinical sample. Studies are split into two sub-groups. The first group of studies reported all ICCs that were calculated, thereby allowing for a relatively unbiased estimate of reliability. The second group of studies selected a subset of calculated ICCs based on the magnitude of the ICC or another nonindependent statistic, and then only reported ICCs from that subset. This practice leads to inflated reliability 
As expected, the meta-analysis of 13 substudies that only reported ICCs above a minimum

260 threshold (average $\mathrm{N}=24.2$, median $\mathrm{N}=18)$ revealed a higher meta-analytic ICC of $.705(95 \% \mathrm{CI}, .628$ -

$\left.261.768 ; \mathrm{P}<.001 ; \mathrm{I}^{2}=17.9\right)$. This estimate, which is 1.78 times the size of the estimate from un-thresholded

262 ICCs, is in the good range, suggesting that the practice of thresholding inflates estimates of reliability in

263 task-fMRI. There was no evidence of between-study heterogeneity $\left(\mathrm{I}^{2}=17.9 ; \mathrm{P}=0.54\right)$.

264 A moderator analysis of all substudies revealed significantly higher reliability for studies that

265 thresholded based on ICC $\left(\mathrm{Q}_{\mathrm{M}}=6.531 \mathrm{df}=1, \mathrm{P}=.010 ; \beta=.140\right)$. In addition, ROIs located in the cortex

266 had significantly higher ICCs than those located in the subcortex $\left(\mathrm{Q}_{\mathrm{M}}=114.476, \mathrm{df}=1, \mathrm{P}<.001 ; \beta=.259\right)$.

267 However, we did not find evidence that the meta-analytic estimate was moderated by task type, task design,

268 task length, test-retest interval, ROI type, sample type, or number of citations per year. Finally, we tested

269 for publication bias using the Egger random effects regression test (Egger et al., 1997) and found no

270 evidence for bias $(\mathrm{Z}=.707, \mathrm{P}=.480)$.

The results of the meta-analysis were illuminating, but not without interpretive difficulty. First, the

272 reliability estimates came from a wide array of tasks and samples, so a single meta-analytical reliability

273 estimate could obscure truly reliable task-fMRI paradigms. Second, the studies used different (and some,

274 now outdated) scanners and different pre-processing and analysis pipelines, leaving open the possibility

275 that reliability has improved with more advanced technology and consistent practices. To address these

276 limitations and possibilities, we conducted pre-registered analyses of two new datasets, using state-of-the-

277 art scanners and practices to assess individual differences in commonly used tasks tapping a variety of

278 cognitive and affective functions.

\section{Reliability of Individual Differences in Task-fMRI: Pre-registered Analyses in Two New Datasets}

We evaluated test-retest reliabilities of activation in a priori regions of interest for 11 commonly 
284 targeting emotion, reward, executive function, motor, language, social cognition, and relational processing.

285 This sample size was determined by the publicly available data in the HCP. In the Dunedin Study, 20

286 participants were scanned twice using a 3T Siemens Skyra, on average 79 days apart ( $\mathrm{sd}=10.3$ days), using

287 four tasks targeting emotion, reward, executive control, and episodic memory. This sample size corresponds

288 to the average sample size used in the meta-analyzed studies. Three of the tasks were similar across the two

289 studies, allowing us to test the replicability of task-fMRI reliabilities. For each of the eight unique tasks

290 across the two studies, we identified the task's primary target region, resulting in a total of eight a priori

291 ROIs (see Methods).

292 Group-level activation. To ensure that the 11 tasks were implemented and processed correctly, we

293 calculated the group-level activation in the target ROIs using the primary contrast of interest for each task

294 (see Supplemental Methods for details). These analyses revealed that each task elicited the expected robust

295 activation in the target ROI at the group level (i.e., across all subjects and sessions; see warm-colored maps

296 in Fig. 4 for the three tasks in common between the two studies and Supplemental Fig. S4 for remaining

297 tasks). 


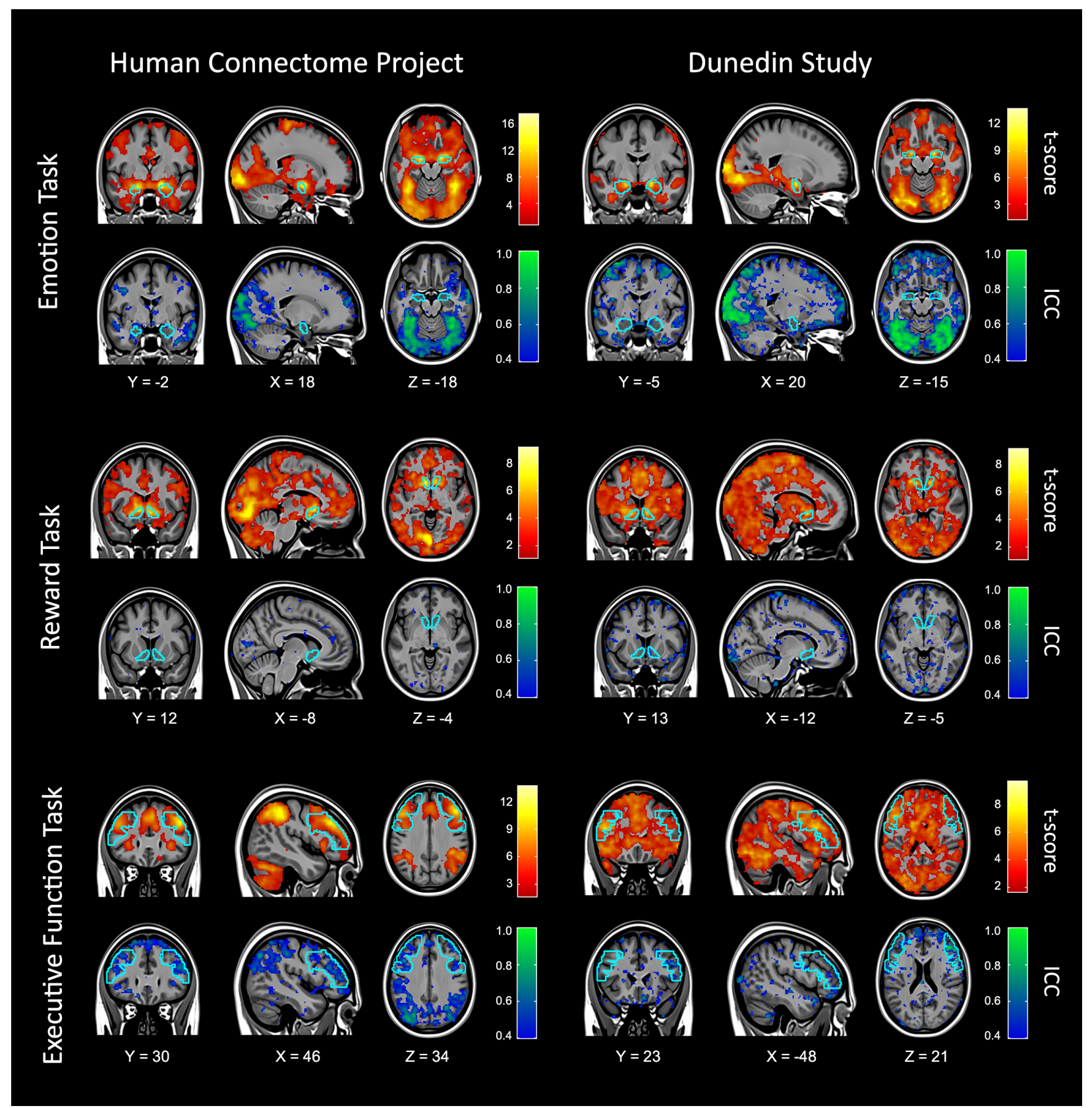

Fig. 4. Whole-brain activation and reliability maps for three task-fMRI measures used in both the Human Connectome Project and Dunedin Study. For each task, a whole-brain activation map of the primary withinsubject contrast (t-score) is displayed in warm colors (top) and a whole-brain map of the between-subjects reliability (ICC) is shown in cool colors (bottom). For each task, the target ROI is outlined in sky-blue. The activation maps are thresholded at $\mathrm{p}<.05$ whole-brain corrected for multiple comparisons using thresholdfree cluster enhancement (Smith \& Nichols, 2009). The ICC maps are thresholded so that voxels with ICC $<.4$ are not colored. These images illustrate that despite robust within-subjects whole-brain activation produced by each task, there is poor between-subjects reliability in this activation, not only in the target ROI but across the whole-brain.

Reliability of regional activation. We investigated the reliability of task activation in both datasets

311 using four steps. First, we tested the reliability of activation in the target ROI for each task. Second, for 
each task we also evaluated the reliability of activation in the other seven a priori ROIs. This was done to

313 test if the reliability of target ROIs was higher than the reliability of activation in other ("non-target") brain

314 regions and to identify any tasks or regions with consistently high reliability. Third, we re-estimated

315 reliability using activation in the left and right hemispheres separately to test if the estimated reliability was

316 harmed by averaging across the hemispheres. Fourth, we tested if the reliability depended on whether ROIs

317 were defined structurally (i.e., using an anatomical atlas) or functionally (i.e., using a set of voxels based

318 on the location of peak activity). See Supplemental Fig. S5 for ICCs of behavior during each fMRI task.

estimates circled in black in Fig. 5, across the seven fMRI tasks, activation in anatomically defined target
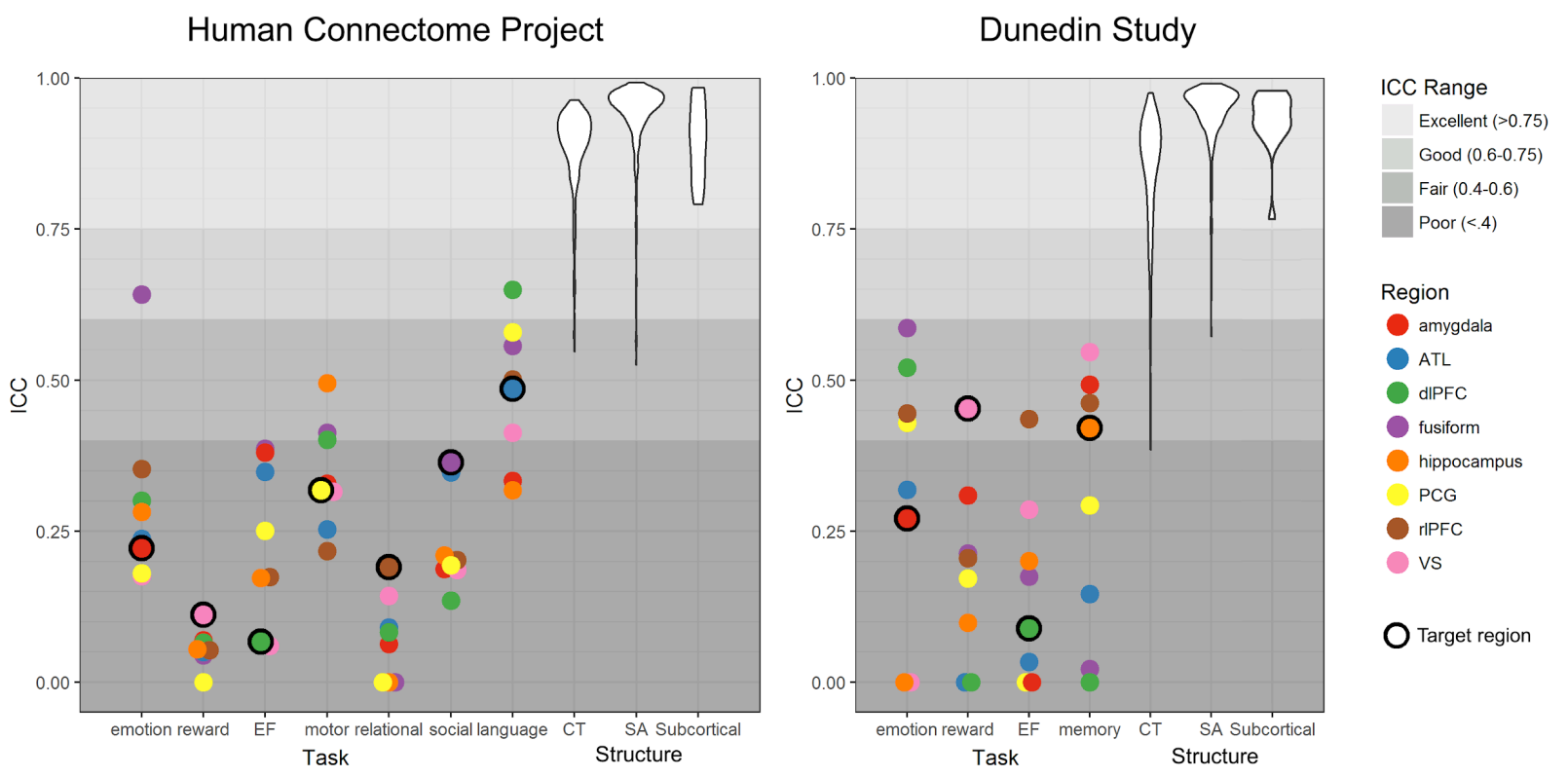

Fig. 5. Test-retest reliabilities of region-wise activation measures in 11 commonly used task-fMRI paradigms ( $\mathrm{EF}=$ executive function). For each task, ICCs were estimated for activation in the a priori target ROI (circled in black) and non-target ROIs selected from the other tasks. These plots show that task-fMRI measures of regional activation in both the Human Connectome Project and Dunedin Study are generally unreliable and the ROIs that are "targeted" by the task are rarely more reliable than non-target ROIs (ATL $=$ anterior temporal lobe, $\mathrm{dlPFC}=$ dorsolateral prefrontal cortex, $\mathrm{PCG}=$ precentral gyrus, $\mathrm{rlPFC}=$ rostrolateral prefrontal cortex, VS = ventral striatum). As a benchmark, ICCs of three common structural MRI measures $(\mathrm{CT}=$ Cortical Thickness, $\mathrm{SA}=$ Surface Area, and Subcortical Volume) are depicted as violin plots representing the distribution of ICCs for each of the 360 parcels for CT and SA, and 17 subcortical structures for grey matter volume. Note that negative ICCs are set to 0 for visualization. 
334

335

336

337

Second, the reliability of task activation in non-target ROIs was also low (Fig. 5; mean ICC =.239; $95 \%$ CI, .188 - .289), but not significantly lower than the reliability in target ROIs $(\mathrm{P}=.474)$.

Third, the reliability of task activation calculated from left and right ROIs separately resembled estimates from averaged ROIs (mean left ICC $=.207$ in target ROIs and .196 in non-target ROIs, mean right ICC $=.259$ in target ROIs and .236 in non-target ROIs; Supplemental Fig. S6).

Fourth, the reliability of task activation in functionally defined ROIs was also low (mean ICC $=$ $.381 ; 95 \%$ CI, $.317-.446)$, with only the motor and social tasks exhibiting ICCs greater than .4 (ICCs $=$ .550 and .446 respectively; see Supplemental Fig. S6).

As an additional step, to account for the family structure present in the HCP, we re-estimated reliability after removing one of each sibling/twin pair in the test-retest sample. Reliability in bilateral anatomical ROIs in the subsample of $\mathrm{N}=26$ unrelated individuals yielded reliabilities very similar to the overall sample (mean ICC = .301 in target ROIs and .218 in non-target ROIs; Supplemental Fig. S6).

Reliability of regional activation in the Dunedin Study. First, as shown by the estimates circled in black in Fig. 5, activation in the anatomically defined target ROI for each of the four tasks had low reliability (mean ICC $=.309 ; 95 \% \mathrm{CI}, .145-.472)$, with no ICCs reaching the "good" range (ICC > .6).

Second, the reliability of activation in the non-target ROIs was also low (Fig. 5; mean ICC $=.193$; 95\% CI, .100 - .286), but not significantly lower than the reliability in target ROIs $(\mathrm{P}=.140)$.

Third, the reliability of task activation calculated for the left and right hemispheres separately was similar to averaged ROIs (mean left ICC $=.243$ in target ROIs and .202 in non-target ROIs, mean right ICC $=.358$ in target ROIs and .192 in non-target ROIs; Supplemental Fig. S6).

Fourth, functionally defined ROIs again did not meaningfully improve reliability (mean ICC $=$ $.325 ; 95 \%$ CI, .197 - .453; see Supplemental Fig. S6).

Reliability of structural measures. To provide a benchmark for evaluating the test-retest reliability of task-fMRI, we investigated the reliability of three commonly used structural MRI measures: cortical 
thickness, surface area and subcortical grey matter volume. Consistent with prior evidence (Han et al., 2006;

361 Maclaren et al., 2014) that structural MRI phenotypes have excellent reliability (i.e., ICCs > .9), global and

362 regional structural MRI measures in the present samples demonstrated very high test-retest reliabilities (Fig.

363 5). For average cortical thickness, ICCs were .953 and .939 in the HCP and Dunedin Study datasets,

364 respectively. In the HCP, parcel-wise (i.e., regional) cortical thickness reliabilities averaged .886 (range

$365.547-.964)$, with $100 \%$ crossing the "fair" threshold, $98.6 \%$ the "good" threshold, and $94.2 \%$ the "excellent"

366 threshold. In the Dunedin Study, parcel-wise cortical thickness reliabilities averaged .846 (range .385 -

$367.975)$, with $99.7 \%$ of ICCs above the "fair" threshold, $96.4 \%$ above "good", and $84.7 \%$ above "excellent."

368 For total surface area, ICCs were .999 and .996 in the HCP and Dunedin Study datasets, respectively. In

369 the HCP, parcel-wise surface area ICCs averaged .937 (range $.526-.992$ ), with $100 \%$ crossing the "fair"

370 threshold, 98.9\% crossing the "good" threshold, and 96.9\% crossing the "excellent" threshold. In the

371 Dunedin Study, surface area ICCs averaged .942 (range .572 - .991), with 100\% above the "fair" threshold,

372 99.7\% above "good," and 98.1\% above "excellent." For subcortical volumes, ICCs in the HCP averaged

373.903 (range .791 - .984), with all ICCs above the "excellent" threshold. In the Dunedin Study, subcortical

374 volumes averaged .931 (range .767 - .979), with all ICCs above the "excellent" threshold. See Supplemental

375 Table S3 for reliabilities of each subcortical region evaluated.

\section{Discussion}

We found evidence that commonly used task-fMRI measures generally do not have the test-retest

reliability necessary for biomarker discovery or brain-behavior mapping. Our meta-analysis of task-fMRI

381 required for good reliability $(\mathrm{ICC}=.6($ Cicchetti \& Sparrow, 1981) $)$ and far below the recommended cutoffs

382 for clinical application (ICC $=.8$ ) or individual-level interpretation (ICC = .9) (Guilford, 1946). Of course,

383 not all task-fMRI measures are the same, and it is not possible to assign a single reliability estimate to all 
individual-difference measures gathered in fMRI research. However, we found little evidence that task type, task length, or test-retest interval had an appreciable impact on the reliability of task-fMRI.

We additionally evaluated the reliability of 11 commonly used task-fMRI measures in the HCP and

387 Dunedin Study. Unlike many of the studies included in our meta-analysis, these two studies were completed

388 recently on modern scanners using cutting-edge acquisition parameters, up-to-date artifact reduction, and

389 state-of-the-art preprocessing pipelines. Regardless, the average test-retest reliability was again poor (ICC

$390=.228$ ). In these analyses, we found no evidence that ROIs "targeted" by the task were more reliable than

391 other, non-target ROIs (mean ICC $=.270$ for target, .228 for non-target) or that any specific task or target

392 ROI consistently produced measures with high reliability. Of interest, the reliability estimate from these

393 two studies was considerably smaller than the meta-analysis estimate (meta-analytic ICC $=.397$ ), possibly

394 due to the phenomenon that pre-registered analyses often yield smaller effect sizes than analyses from

395 publications without pre-registration, which affords increased flexibility in analytic decision-making

396 (Schäfer \& Schwarz, 2019).

\section{The two disciplines of fMRI research}

Our results harken back to Lee Cronbach's classic 1957 article in which he described the "two disciplines of scientific psychology" (Cronbach, 1957). According to Cronbach, the "experimental"

401 discipline strives to uncover universal human traits and abilities through experimental control and group

402 averaging, whereas the "correlational" discipline strives to explain variation between people by measuring

403 how they differ from one another. A fundamental distinction between the two disciplines is how they treat

404 individual differences. For the experimental researcher, variation between people is error that must be

405 minimized to detect the largest experimental effect. For the correlational investigator, variation between

406 people is the primary unit of analysis and must be measured carefully to extract reliable individual

407 differences (Cronbach, 1957; Hedge et al., 2018).

408 Current task-fMRI paradigms are largely descended from the "experimental" discipline. Task-

409 fMRI paradigms are intentionally designed to reveal how the average human brain responds to provocation,

410 while minimizing between-subject variance. Paradigms that are able to elicit robust targeted brain activity 
411 at the group-level are subsequently converted into tools for assessing individual differences. Within-subject

412 robustness is, then, often inappropriately invoked to suggest between-subject reliability, despite the fact

413 that reliable within-subject experimental effects at a group level can arise from unreliable between-subjects

414 measurements (Fröhner et al., 2019).

415 This reasoning is not unique to task-fMRI research. Behavioral measures that elicit robust within-

416 subject (i.e., group) effects have been shown to have low between-subjects reliability; for example, the

417 mean test-retest reliability of the Stroop Test $(\mathrm{ICC}=.45$; (Hedge et al., 2018)) is strikingly similar to the

418 mean reliability of our task-fMRI meta-analysis $(\mathrm{ICC}=.397)$. Nor is it the case that MRI measures, or even

419 the BOLD signal itself, are inherently unreliable. Both structural MRI measures in our analyses (see Fig.

420 5), as well as measures of intrinsic functional connectivity estimated from long fMRI scans (Elliott et al.,

4212019 ; Gratton et al., 2018), demonstrate high test-retest reliability. Thus, it is not the tool that is problematic

422 but rather the strategy of adopting tasks developed for experimental cognitive neuroscience that appear to

423 be poorly suited for reliably measuring differences in brain activation between people.

\section{Recommendations and Future Directions}

We next consider several avenues for maximizing the value of existing datasets as well as

427 improving the reliability of task-fMRI moving forward. We begin with recommendations that can be

428 implemented immediately $(1,2)$, before moving on to recommendations that will require additional data

429 collection and innovation $(3,4)$.

\section{1) Immediate opportunities for task-fMRI: from brain hotspots to whole-brain signatures}

432 Currently, the majority of task-fMRI measures are based on contrasts between conditions (i.e.,

433 change scores), extracted from ROIs. However, change scores will always have lower reliability than their

434 constituent measures (Hedge et al., 2018), and have been shown to undermine the reliability of task-fMRI

435 (Infantolino et al., 2018). However, contrast-based activation values extracted from ROIs represent only

436 one possible measure of individual differences that can be derived from task-fMRI data. For example,

437 several multivariate methods have been proposed to increase the reliability and predictive utility of task- 
fMRI measures by exploiting the high dimensionality inherent in fMRI data (Dubois \& Adolphs, 2016;

439 Yarkoni \& Westfall, 2017). To name a few, the reliability of task-fMRI may be improved by developing

440 measures with latent variable models (Cooper et al., 2019), measuring individual differences in

441 representational spaces with multi-voxel pattern analysis (Norman et al., 2006), and training cross-validated

442 machine learning models that establish reliability through prediction of individual differences in

443 independent samples (Yarkoni \& Westfall, 2017). In addition, in many already-collected datasets, task-

444 fMRI can be combined with resting-state fMRI data to produce reliable measures of intrinsic functional

445 connectivity (Elliott et al., 2019; Greene et al., 2018). Thus, there are multiple available approaches to

446 maximizing the value of existing task-fMRI datasets in the context of biomarker discovery and individual

447 differences research.

\section{2) Create a norm of reporting the reliability of task-fMRI measures}

The "replicability revolution" in psychological science (Nosek et al., 2015) provides a timely

451 example of how rapidly changing norms can shape research practices and standards. In just a few years,

452 practices to enhance replicability, like pre-registration of hypotheses and analytic strategies, have risen in

453 popularity (Nosek et al., 2018). We believe similar norms would be beneficial for task-fMRI in the context

454 of biomarker discovery and brain-behavior mapping. In particular, researchers should report the reliabilities

455 for all task-fMRI measures whenever they are used to study individual differences (Parsons et al., 2019).

456 In doing so, however, researchers need to ensure adequate power to evaluate test-retest reliability with

457 confidence. Given that correlations begin to stabilize with around 150 observations (Schönbrodt \& Perugini,

458 2013), our confidence in knowing "the" reliability of any specific task will depend on collecting larger test-

459 retest datasets. We provide evidence that the task-fMRI literature generally has low reliability; however,

460 due to the relatively small size of each test-retest sample reported here, we urge readers to avoid making

461 strong conclusions about the reliability of specific fMRI tasks. In the pursuit of precise reliability estimates,

462 it will be important for researchers to collect larger test-retest samples, explore test-retest moderators (e.g.

463 test-retest interval) and avoid reporting inflated reliabilities that can arise from circular statistical analyses

464 (for detailed recommendations see (Kriegeskorte et al., 2010, 2009; Vul et al., 2009)). 
Researchers can also provide evidence of between-subjects reliability in the form of internal consistency. While test-retest reliability provides an estimate of stability over time that is suited for trait

467 and biomarker research, it is a conservative estimate that requires extra data collection and can be

468 undermined by habituation effects and rapid fluctuations (Hajcak et al., 2017). In some cases, internal

469 consistency will be more practical because it is cheaper, as it does not require additional data collection and

470 can be used in any situation where the task-fMRI measure of interest is comprised of multiple trials

471 (Streiner, 2003). Internal consistency is particularly well-suited for measures that are expected to change

472 rapidly and index transient psychological states (e.g., current emotions or thoughts). However, internal

473 consistency alone is not adequate for prognostic biomarkers. Establishing a norm of explicitly reporting

474 measurement reliability would increase the replicability of task-fMRI findings and accelerate biomarker

475 discovery.

\section{3) More data from more subjects}

Our ability to detect reliable individual differences using task-fMRI will depend, in part, on the

479 field embracing two complementary improvements to the status quo: 1) more subjects per study and 2)

480 more data per subject. It has been suggested that neuroscience is generally an underpowered enterprise, and

481 that small sample sizes undermine fMRI research in particular (Button et al., 2013; Szucs \& Ioannidis,

482 2017). The results presented here suggest that this "power failure" may be further compounded by low

483 reliability in task-fMRI. The median sample size in fMRI research is 28.5 (Poldrack et al., 2017). However,

484 as shown in Fig. 1, task-fMRI measures with ICCs of .397 (the meta-analytic mean reliability) would

485 require $\mathrm{N}>214$ to achieve $80 \%$ power to detect brain-behavior correlations of .3 , a moderate effect size

486 equal to the size of the largest replicated brain-behavior associations (Elliott et al., 2018; Nave et al., 2019).

487 For $\mathrm{r}=.1$ (a small effect size common in psychological research (Funder \& Ozer, 2019)), adequately

488 powered studies require $\mathrm{N}>2,000$. And, these calculations are actually best-case scenarios given that they

489 assume perfect reliability of the second "behavioral" variable (see Figure 1). Increasing the sample size of

490 task-fMRI studies and requiring power analyses that take into account unreliability represent a meaningful

491 way forward for boosting the replicability of individual differences research with task-fMRI. 
Without substantially higher reliability, task-fMRI measures will fail to provide biomarkers that

493 are meaningful on an individual level. One promising method to improve the reliability of fMRI is to collect

494 more data per subject. Increasing the amount of data collected per subject has been shown to improve the

495 reliability of functional connectivity (Elliott et al., 2019; Gratton et al., 2018) and preliminary efforts

496 suggest this may be true for task-fMRI as well (Gordon et al., 2017). Pragmatically, collecting additional

497 fMRI data will be burdensome for participants, especially in children and clinical populations, where longer

498 scan times often result in greater data artifacts particularly from increased motion. Naturalistic fMRI

499 represents one potential solution to this challenge. In naturalistic fMRI, participants watch stimulus-rich

500 movies during scanning instead of completing traditional cognitive neuroscience tasks. Initial efforts

501 suggest that movie watching is highly engaging for subjects, allows more data collection with less motion

502 and may even better elicit individual differences in brain activity by emphasizing ecological validity over

503 experimental control (Vanderwal et al., 2018). As the field launches large-scale neuroimaging studies (e.g.

504 HCP, UK Biobank, ABCD) in the pursuit of brain biomarkers of disease risk, it is critical that we are

505 confident in the psychometric properties of task-fMRI measurements. This will require funders to advocate

506 and support the collection of more data from more subjects.

\section{4) Develop tasks from the ground up to optimize reliable and valid measurement}

Instead of continuing to adopt fMRI tasks from experimental studies emphasizing within-subjects

510 effects, we need to develop new tasks (and naturalistic stimuli) from the ground up with the goal of

511 optimizing their utility in individual differences research (i.e., between-subjects effects). Psychometrics

512 provides many tools and methods for developing reliable individual differences measures that have been

513 underutilized in task-fMRI development. For example, stimuli in task-fMRI could be selected based on

514 their ability to maximally distinguish groups of subjects or to elicit reliable between subject variance. As

515 noted in recommendation 1, psychometric tools for test construction could be adopted to optimize reliable

516 task-fMRI measures including item analysis, latent variable modelling, and internal-consistency measures

517 (Crocker \& Algina, 2006). 


\section{Conclusion}

520 A prominent goal of task-fMRI research has been to identify abnormal brain activity that could aid

521 in the diagnosis, prognosis, and treatment of brain disorders. We find that commonly used task-fMRI

522 measures lack minimal reliability standards necessary for accomplishing this goal. Intentional design and

523 optimization of task-fMRI paradigms are needed to measure reliable variation between individuals. As task-

524 fMRI research faces the challenges of reproducibility and replicability, we draw attention to the importance

525 of reliability as well. In the age of individualized medicine and precision neuroscience, funding is needed

526 for novel task-fMRI research that embraces the psychometric rigor necessary to generate clinically 527 actionable knowledge. 
528

529

530

531

532

533

534

535

536

537

538

\section{Box 1: Why is reliability critical for task-fMRI research?}

Test-retest reliability is widely quantified using the intraclass correlation coefficient (ICC (Shrout \& Fleiss, 1979)). ICC can be thought of as the proportion of a measure's total variance that is accounted for by variation between individuals. An ICC can take on values between -1 and 1, with values approaching 1 indicating nearly perfect stability of individual differences across test-retest measurements, and values at or below 0 indicating no stability. Classical test theory states that all measures are made up of a true score plus measurement error (Novick, 1965). The ICC is used to estimate the amount of reliable, true-score variance present in an individual differences measure. When a measure is taken at two timepoints, the variance in scores that is due to measurement error will consist of random noise and will fail to correlate with itself across test-retest measurements. However, the variance in a score that is due to true score will be stable and correlate with itself across timepoints (Crocker \& Algina, 2006). Measures with ICC $<.40$ are thought to have "poor" reliability, those with ICCs between .40 - .60 "fair" reliability, .60 - .75 "good" reliability, and $>.75$ "excellent" reliability. An ICC $>.80$ is considered a clinically required standard for reliability in psychology (Cicchetti \& Sparrow, 1981).

Reliability is critical for research because the correlation observed between two measures, A and $\mathrm{B}$, is constrained by the square root of the product of each measure's reliability (Nunnally, 1959):

$$
r\left(A_{\text {observed }}, B_{\text {observed }}\right)=r\left(A_{\text {true }}, B_{\text {true }}\right) * \sqrt{\text { reliability }\left(A_{\text {observed }}\right) * \text { reliability }\left(B_{\text {observed }}\right)}
$$

Low reliability of a measure reduces statistical power and increases the sample size required to detect a correlation with another measure. Fig. 1 shows sample sizes required for $80 \%$ power to detect correlations between aa task-fMRI measure of individual differences in brain activation and a behavioral/clinical phenotype, across a range of reliabilities of the task-fMRI measure and expected effect sizes. Power curves are given for three levels of reliability of the hypothetical behavioral/clinical phenotype, where the first two panels (behavioral ICC $=.6$ and .8 ) represent most typical scenarios. 
A.C., A.R.H., T.E.M, M.L.E., and A.R.K. conceived the study and data analysis plan. M.L.E.,

the results and contributed to the revision of the manuscript.

\section{Acknowledgments}

564 (Principal Investigators: David Van Essen and Kamil Ugurbil; 1U54MH091657) funded by the 16 NIH

565 Institutes and Centers that support the NIH Blueprint for Neuroscience Research; and by the McDonnell

566 Center for Systems Neuroscience at Washington University.

568 The Dunedin Study is supported by NIA grants R01AG049789 and R01AG032282 and U.K. Medical

569 Research Council grant P005918. The Dunedin Multidisciplinary Health and Development Research Unit

570 is supported by the New Zealand Health Research Council and the New Zealand Ministry of Business,

571 Innovation and Employment (MBIE). MLE is supported by the National Science Foundation Graduate

572 Research Fellowship under Grant No. NSF DGE-1644868. Thanks to the members of the Advisory Board

573 for the Dunedin Neuroimaging Study. The authors would also like to thank Tim Strauman and Ryan

574 Bogdan for their feedback on an initial draft of this manuscript, as well as extensive feedback from peer

575 reviewers. 


\section{References}

577 Barch, D. M., Burgess, G. C., Harms, M. P., Petersen, S. E., Schlaggar, B. L., Corbetta, M., Glasser, M.

F., Curtiss, S., Dixit, S., Feldt, C., Nolan, D., Bryant, E., Hartley, T., Footer, O., Bjork, J. M., $80,169-189$.

Bennett, C. M., \& Miller, M. B. (2010). How reliable are the results from functional magnetic resonance imaging? Annals of the New York Academy of Sciences, 1191, 133-155.

Binder, J. R., Gross, W. L., Allendorfer, J. B., Bonilha, L., Chapin, J., Edwards, J. C., Grabowski, T. J., Langfitt, J. T., Loring, D. W., Lowe, M. J., Koenig, K., Morgan, P. S., Ojemann, J. G., Rorden, C., Szaflarski, J. P., Tivarus, M. E., \& Weaver, K. E. (2011). Mapping anterior temporal lobe language areas with fMRI: a multicenter normative study. NeuroImage, 54(2), 1465-1475.

Borenstein, M., Hedges, L. V., Higgins, J. P. T., \& Rothstein, H. R. (2009). Introduction to MetaAnalysis. https://doi.org/10.1002/9780470743386

Button, K. S., Ioannidis, J. P. A., Mokrysz, C., Nosek, B. A., Flint, J., Robinson, E. S. J., \& Munafò, M. R. (2013). Power failure: why small sample size undermines the reliability of neuroscience. Nature Reviews. Neuroscience, 14(5), 365-376.

Chen, G., Saad, Z. S., Britton, J. C., Pine, D. S., \& Cox, R. W. (2013). Linear mixed-effects modeling approach to FMRI group analysis. NeuroImage, 73, 176-190.

Chen, G., Taylor, P. A., Haller, S. P., Kircanski, K., Stoddard, J., Pine, D. S., Leibenluft, E., Brotman, M.

601 Cooper, S. R., Jackson, J. J., Barch, D. M., \& Braver, T. S. (2019). Neuroimaging of individual A., \& Cox, R. W. (2018). Intraclass correlation: Improved modeling approaches and applications for neuroimaging. Human Brain Mapping, 39(3), 1187-1206.

Cicchetti, D. V., \& Sparrow, S. A. (1981). Developing criteria for establishing interrater reliability of specific items: applications to assessment of adaptive behavior. American Journal of Mental Deficiency, 86(2), 127-137. differences: A latent variable modeling perspective. In Neuroscience \& Biobehavioral Reviews (Vol. 
98, pp. 29-46). https://doi.org/10.1016/j.neubiorev.2018.12.022

604 Crocker, L., \& Algina, J. (2006). Introduction to Classical and Modern Test Theory. Wadsworth

605 Publishing Company.

606 Cronbach, L. J. (1957). The two disciplines of scientific psychology. In American Psychologist (Vol. 12,

$607 \quad$ Issue 11, pp. 671-684). https://doi.org/10.1037/h0043943

608 Delgado, M. R., Nystrom, L. E., Fissell, C., Noll, D. C., \& Fiez, J. A. (2000). Tracking the hemodynamic responses to reward and punishment in the striatum. Journal of Neurophysiology, 84(6), 3072-3077.

Drobyshevsky, A., Baumann, S. B., \& Schneider, W. (2006). A rapid fMRI task battery for mapping of visual, motor, cognitive, and emotional function. NeuroImage, 31(2), 732-744.

Dubois, J., \& Adolphs, R. (2016). Building a Science of Individual Differences from fMRI. Trends in Cognitive Sciences, 20(6), 425-443.

Egger, M., Davey Smith, G., Schneider, M., \& Minder, C. (1997). Bias in meta-analysis detected by a simple, graphical test. $B M J, 315(7109), 629-634$.

Elliott, M. L., Belsky, D. W., Anderson, K., Corcoran, D. L., Ge, T., Knodt, A., Prinz, J. A., Sugden, K., Williams, B., Ireland, D., Poulton, R., Caspi, A., Holmes, A., Moffitt, T., \& Hariri, A. R. (2018). A

624 Fröhner, J. H., Teckentrup, V., Smolka, M. N., \& Kroemer, N. B. (2019). Addressing the reliability Cortex . https://doi.org/10.1093/cercor/bhy219

Elliott, M. L., Knodt, A. R., Cooke, M., Kim, M. J., Melzer, T. R., Keenan, R., Ireland, D., Ramrakha, S., Poulton, R., Caspi, A., Moffitt, T. E., \& Hariri, A. R. (2019). General functional connectivity: Shared features of resting-state and task fMRI drive reliable and heritable individual differences in 
Glasser, M. F., Coalson, T. S., Robinson, E. C., Hacker, C. D., Harwell, J., Yacoub, E., Ugurbil, K., Andersson, J., Beckmann, C. F., Jenkinson, M., Smith, S. M., \& Van Essen, D. C. (2016). A multimodal parcellation of human cerebral cortex. Nature, 536(7615), 171-178.

Gordon, E. M., Laumann, T. O., Gilmore, A. W., Newbold, D. J., Greene, D. J., Berg, J. J., Ortega, M., Hoyt-Drazen, C., Gratton, C., Sun, H., Hampton, J. M., Coalson, R. S., Nguyen, A. L., McDermott, K. B., Shimony, J. S., Snyder, A. Z., Schlaggar, B. L., Petersen, S. E., Nelson, S. M., \& Dosenbach,

Gratton, C., Laumann, T. O., Nielsen, A. N., Greene, D. J., Gordon, E. M., Gilmore, A. W., Nelson, S. M., Coalson, R. S., Snyder, A. Z., Schlaggar, B. L., Dosenbach, N. U. F., \& Petersen, S. E. (2018). Functional Brain Networks Are Dominated by Stable Group and Individual Factors, Not Cognitive or Daily Variation. Neuron, 98(2), 439-452.e5.

Greene, A. S., Gao, S., Scheinost, D., \& Constable, R. T. (2018). Task-induced brain state manipulation improves prediction of individual traits. Nature Communications, 9(1), 2807.

Guilford, J. P. (1946). New Standards For Test Evaluation. In Educational and Psychological

Hajcak, G., Meyer, A., \& Kotov, R. (2017). Psychometrics and the neuroscience of individual differences: Internal consistency limits between-subjects effects. Journal of Abnormal Psychology,

Han, X., Jovicich, J., Salat, D., van der Kouwe, A., Quinn, B., Czanner, S., Busa, E., Pacheco, J., Albert, M., Killiany, R., Maguire, P., Rosas, D., Makris, N., Dale, A., Dickerson, B., \& Fischl, B. (2006). Reliability of MRI-derived measurements of human cerebral cortical thickness: the effects of field produce reliable individual differences. Behavior Research Methods, 50(3), 1166-1186. 
Herting, M. M., Gautam, P., Chen, Z., Mezher, A., \& Vetter, N. C. (2018). Test-retest reliability of longitudinal task-based fMRI: Implications for developmental studies. Developmental Cognitive Neuroscience, 33, 17-26.

Infantolino, Z. P., Luking, K. R., Sauder, C. L., Curtin, J. J., \& Hajcak, G. (2018). Robust is not

Knutson, B., Westdorp, A., Kaiser, E., \& Hommer, D. (2000). FMRI visualization of brain activity during a monetary incentive delay task. NeuroImage, 12(1), 20-27.

Kriegeskorte, N., Lindquist, M. A., Nichols, T. E., Poldrack, R. A., \& Vul, E. (2010). Everything you never wanted to know about circular analysis, but were afraid to ask. Journal of Cerebral Blood

Kriegeskorte, N., Simmons, W. K., Bellgowan, P. S. F., \& Baker, C. I. (2009). Circular analysis in systems neuroscience: the dangers of double dipping. Nature Neuroscience, 12(5), 535-540.

Kwong, K. K., Belliveau, J. W., Chesler, D. A., Goldberg, I. E., Weisskoff, R. M., Poncelet, B. P., Flow and Metabolism: Official Journal of the International Society of Cerebral Blood Flow and Kennedy, D. N., Hoppel, B. E., Cohen, M. S., \& Turner, R. (1992). Dynamic magnetic resonance imaging of human brain activity during primary sensory stimulation. Proceedings of the National Academy of Sciences of the United States of America, 89(12), 5675-5679.

Logothetis, N. K., Pauls, J., Augath, M., Trinath, T., \& Oeltermann, A. (2001). Neurophysiological investigation of the basis of the fMRI signal. Nature, 412(6843), 150-157.

Maclaren, J., Han, Z., Vos, S. B., Fischbein, N., \& Bammer, R. (2014). Reliability of brain volume measurements: a test-retest dataset. Scientific Data, 1, 140037.

Manuck, S. B., Brown, S. M., Forbes, E. E., \& Hariri, A. R. (2007). Temporal stability of individual differences in amygdala reactivity. The American Journal of Psychiatry, 164(10), 1613-1614.

McGraw, K. O., \& Wong, S. P. (1996). Forming inferences about some intraclass correlation coefficients. In Psychological Methods (Vol. 1, Issue 1, pp. 30-46). https://doi.org/10.1037//1082-989x.1.1.30 

aCC-BY 4.0 International license.

Nave, G., Jung, W. H., Karlsson Linnér, R., Kable, J. W., \& Koellinger, P. D. (2019). Are Bigger Brains Smarter? Evidence From a Large-Scale Preregistered Study. Psychological Science, 30(1), 43-54.

Nord, C. L., Gray, A., Charpentier, C. J., Robinson, O. J., \& Roiser, J. P. (2017). Unreliability of putative fMRI biomarkers during emotional face processing. NeuroImage, 156, 119-127.

Norman, K. A., Polyn, S. M., Detre, G. J., \& Haxby, J. V. (2006). Beyond mind-reading: multi-voxel pattern analysis of fMRI data. Trends in Cognitive Sciences, 10(9), 424-430.

Nosek, B. A., Alter, G., Banks, G. C., Borsboom, D., Bowman, S. D., Breckler, S. J., Buck, S., Chambers, C. D., Chin, G., Christensen, G., Contestabile, M., Dafoe, A., Eich, E., Freese, J., Glennerster, R.,

Nosek, B. A., Ebersole, C. R., DeHaven, A. C., \& Mellor, D. T. (2018). The preregistration revolution. Proceedings of the National Academy of Sciences of the United States of America, 115(11), 26002606.

Novick, M. R. (1965). THE AXIOMS AND PRINCIPAL RESULTS OF CLASSICAL TEST THEORY. In ETS Research Bulletin Series (Vol. 1965, Issue 1, pp. i - 31). https://doi.org/10.1002/j.23338504.1965.tb00132.x

Nunnally, J. C. (1959). Introduction to Psychological Measurement.

Parsons, S., Kruijt, A.-W., \& Fox, E. (2019). Psychological Science Needs a Standard Practice of Reporting the Reliability of Cognitive-Behavioral Measurements. In Advances in Methods and Practices in Psychological Science (Vol. 2, Issue 4, pp. 378-395). https://doi.org/10.1177/2515245919879695

Peterson, B. S., Skudlarski, P., Gatenby, J. C., Zhang, H., Anderson, A. W., \& Gore, J. C. (1999). An fMRI study of Stroop word-color interference: evidence for cingulate subregions subserving multiple distributed attentional systems. Biological Psychiatry, 45(10), 1237-1258.

Plichta, M. M., Schwarz, A. J., Grimm, O., Morgen, K., Mier, D., Haddad, L., Gerdes, A. B. M., Sauer, C., Tost, H., Esslinger, C., Colman, P., Wilson, F., Kirsch, P., \& Meyer-Lindenberg, A. (2012). Test- 
retest reliability of evoked BOLD signals from a cognitive-emotive fMRI test battery. NeuroImage, 60(3), 1746-1758.

713 Poldrack, R. A., Baker, C. I., Durnez, J., Gorgolewski, K. J., Matthews, P. M., Munafò, M. R., Nichols, T.

714 E., Poline, J.-B., Vul, E., \& Yarkoni, T. (2017). Scanning the horizon: towards transparent and

715 reproducible neuroimaging research. Nature Reviews. Neuroscience, 18(2), 115-126.

716 Poulton, R., Moffitt, T. E., \& Silva, P. A. (2015). The Dunedin Multidisciplinary Health and

717 Development Study: overview of the first 40 years, with an eye to the future. Social Psychiatry and $718 \quad$ Psychiatric Epidemiology, 50(5), 679-693.

719 Schäfer, T., \& Schwarz, M. A. (2019). The Meaningfulness of Effect Sizes in Psychological Research:

Schönbrodt, F. D., \& Perugini, M. (2013). At what sample size do correlations stabilize? In Journal of

724 Shrout, P. E., \& Fleiss, J. L. (1979). Intraclass correlations: uses in assessing rater reliability. Psychological Bulletin, 86(2), 420-428.

726 Smith, R., Keramatian, K., \& Christoff, K. (2007). Localizing the rostrolateral prefrontal cortex at the 727 individual level. NeuroImage, 36(4), 1387-1396.

728 Smith, S., \& Nichols, T. (2009). Threshold-free cluster enhancement: Addressing problems of smoothing, threshold dependence and localisation in cluster inference. In NeuroImage (Vol. 44, Issue 1, pp. 8398). https://doi.org/10.1016/j.neuroimage.2008.03.061

Streiner, D. L. (2003). Starting at the beginning: an introduction to coefficient alpha and internal consistency. Journal of Personality Assessment, 80(1), 99-103. vulnerability to future life stress. Neuron, 85(3), 505-511.

735 Szucs, D., \& Ioannidis, J. P. A. (2017). Empirical assessment of published effect sizes and power in the 736 recent cognitive neuroscience and psychology literature. PLoS Biology, 15(3), e2000797.

737 Vanderwal, T., Eilbott, J., \& Castellanos, F. X. (2018). Movies in the magnet: Naturalistic paradigms in 
developmental functional neuroimaging. Developmental Cognitive Neuroscience, 100600.

Van Essen, D. C., Smith, S. M., Barch, D. M., Behrens, T. E. J., Yacoub, E., Ugurbil, K., \& WU-Minn HCP Consortium. (2013). The WU-Minn Human Connectome Project: an overview. NeuroImage, $80,62-79$.

Vul, E., Harris, C., Winkielman, P., \& Pashler, H. (2009). Puzzlingly High Correlations in fMRI Studies of Emotion, Personality, and Social Cognition. Perspectives on Psychological Science: A Journal of

Wheatley, T., Milleville, S. C., \& Martin, A. (2007). Understanding animate agents: distinct roles for the the Association for Psychological Science, 4(3), 274-290. social network and mirror system. Psychological Science, 18(6), 469-474.

Woo, C.-W., Chang, L. J., Lindquist, M. A., \& Wager, T. D. (2017). Building better biomarkers: brain models in translational neuroimaging. Nature Neuroscience, 20(3), 365-377.

752 Yarkoni, T., \& Westfall, J. (2017). Choosing Prediction Over Explanation in Psychology: Lessons From

753 Machine Learning. Perspectives on Psychological Science: A Journal of the Association for

$754 \quad$ Psychological Science, 12(6), 1100-1122.

755 Zeineh, M. M., Engel, S. A., Thompson, P. M., \& Bookheimer, S. Y. (2003). Dynamics of the 756 hippocampus during encoding and retrieval of face-name pairs. Science, 299(5606), 577-580. 